\title{
Chronic idiopathic thrombocytopenic purpura: incidence, treatment, and outcome
}

\author{
M M Reid
}

\begin{abstract}
In order to determine incidence, outcome, trends in management and natural history, data on 92 children with chronic idiopathic thrombocytopenic purpura (ITP), comprising 66 from a single centre's experience between 1950 and 1980 and all 26 presenting from a defined population between 1984 and 1994, have been analysed. Its incidence, calculated from the population based group, is $0 \cdot 46 / 10^{5}$ children per year. Twenty nine of $34(85 \%)$ remitted after splenectomy. Short initial histories predicted response to splenectomy. Splenectomy was offered only half as frequently in the last $\mathbf{1 0}$ years as in the 30 year, single centre group of children. Most (39 cases) of those not offered or successfully treated by splenectomy recovered spontaneously. The predicted spontaneous remission rate in 85 with adequate follow up data is $61 \%$ after 15 years. No other form of active treatment was of lasting benefit. No death solely attributable to chronic ITP occurred. The high spontaneous recovery rate, low mortality, and generally benign outcome may encourage a less interventionist approach to management.
\end{abstract}

(Arch Dis Child 1995; 72: 125-128)

Keywords: thrombocytopenia, purpura, chronic.

Chronic idiopathic thrombocytopenic purpura (ITP) is a rare disease, developing in a minority of children with ITP, but its management is fraught with worry and controversy. Numerous reviews and the large number of therapeutic approaches attest to uncertainty about the most effective and safe treatment and highlight the lack of knowledge about its natural history. ${ }^{1-5}$ In Lilleyman's words, we remain 'transfixed by the fear of intracranial haemorrhage'.5

Walker and Walker reported their experience of all cases of ITP presenting to a single centre between 1950 and $1980 .^{6}$ However, those with disease persisting beyond six months, the current, arbitrary definition of chronic ITP, were not singled out for analysis as a distinct group. This report analyses data on those with chronic ITP from that series together with all new such cases presenting from a defined population in a 10 year period, in order to calculate its incidence, document outcome and, where possible, its natural history, and note any trends in approach to management. The aim is to provide background data which may, when added to the various published opinions about management, be useful in formulating or modifying plans of management for individual children with chronic ITP.

\section{Methods}

Two data sets have been used. The first comprises data on all cases of chronic ITP seen in the Royal Victoria Infirmary between 1950 and 1980, extracted from the series described by Walker and Walker. ${ }^{6}$ This represents a single centre, rather than a demographically defined, group. The second set comprises all recognised cases of chronic ITP in the Northern health region of the UK (excluding Barrow) arising between June 1984 and May 1994. Cases were collected prospectively but, in addition, a further search was carried out through the network of the Northern Region Consultant Haematologists' Group, to identify cases not already known. Many had been referred to this centre; details of others were obtained from individual haematologists and paediatricians.

Standard diagnostic criteria, comprising isolated thrombocytopenia with a history of purpura and absence of other causes, were used. Chronic ITP was defined as thrombocytopenia (platelet count $<150 \times 10^{9} / 1$ ) persisting more than six months after initial diagnosis of ITP. Recovery was defined as absence of purpura or abnormal bruising together with a persistently normal platelet count.

Incidence was calculated from the more recent, demographically defined, data set using population estimates from the Office of Population Censuses and Surveys and the Northern Regional Health Authority.

Rates of spontaneous recovery and response to splenectomy from the combined data sets were related to the duration of symptoms before initial presentation. The arbitrary division by Walker and Walker, ${ }^{6}$ into those with initial symptoms for up to two weeks and the remainder, has been retained. For the purpose of these comparisons, children 'at risk' of spontaneous recovery comprise all those who did not have a splenectomy together with those whom it failed to cure. Comparison of response rates to splenectomy was by Fisher's exact test, and of spontaneous recovery rates by $\chi^{2}$ with Yates's correction.

Probability of spontaneous remission was calculated by the Kaplan-Meier life table method. Successful splenectomies and deaths were censored. For simplicity, and because of uncertainty about precise timing of remission, events and censoring were grouped into single 


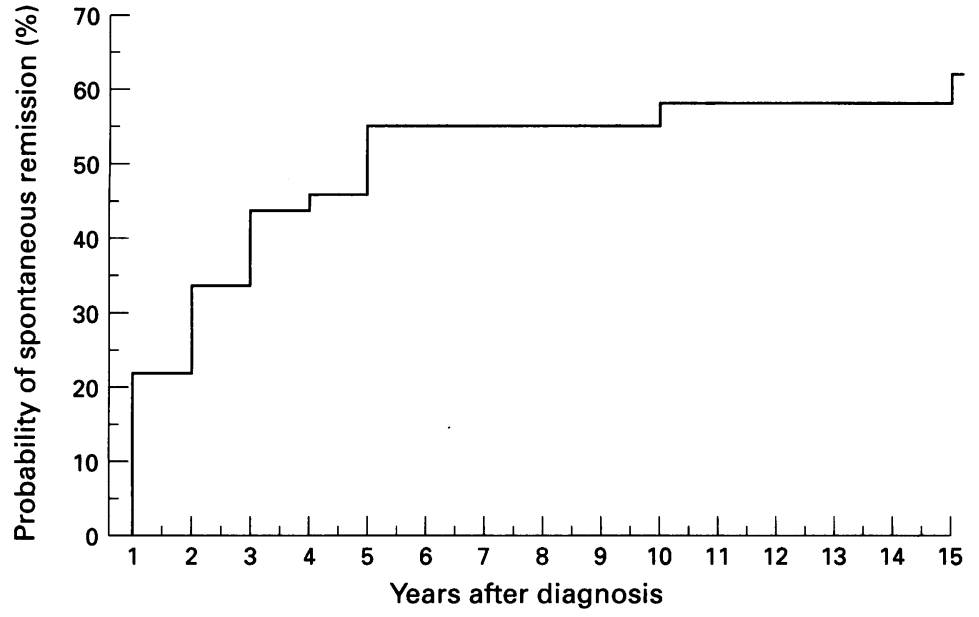

No at risk : $8559 \quad 46 \quad 34 \quad 27 \quad 25$

15

12

Probability of spontaneous remission in 85 cases with adequate follow up data. Cases were censored at successful splenectomy, or death. One spontaneous remission occurred among the seven cases not included from data set 1 . One late spontaneous remission (at 19 years) is also not shown because of uncertainty of the number at risk beyond 15 years.

years for the first five years and then into two five year periods.

\section{Results}

NUMBERS OF CASES

The first data set (extracted from Walker and Walker ${ }^{6}$ ) comprised 66 children, the second 26 children with chronic ITP. In this second group there were 13 girls and 13 boys, with ages ranging between 1 and 14 years at initial presentation. Twenty one of this group were between 1 and 8 years old (median 4 years), four were aged 13 and one 14 years. Fifteen of the more recent group had been centrally referred for advice or management and 11 were identified through the subsequent search. One child with ITP, successfully treated by splenectomy, has not been included because splenectomy was carried out four months after presenting with ITP. Bone marrow was examined in all but five cases in the more recent group and was normal in each.

\section{INCIDENCE}

The population of children aged $<15$ years in the Northern health region (excluding Barrow) between 1985 and 1992 varied between 564000 and 586000 , out of which arose the 26 cases in the second data set, giving an incidence of chronic ITP of approximately 0.46 cases $/ 10^{5}$ children per year.

\section{TREATMENT AND OUTCOME}

Splenectomy was the only active treatment which resulted in complete remission. Other options are considered below under the section 'Effects of treatment other than splenectomy'. Of the 66 in data set $1,32(48 \%)$ underwent splenectomy, of whom 24 remitted, whereas this treatment was offered to only five of the 26 $(19 \%)$ in data set 2 , all of whom remitted. The two groups are not strictly comparable because some clinically mild cases may have escaped inclusion in the first group simply by not being referred. However, there does now seem to be more reluctance to remove spleens than during the 30 years covered by the earlier study.

Spontaneous remissions had occurred in 27 of the 42 children at risk in the first data set at the time of its publication, ${ }^{6}$ and in one further patient since, and in 11 of the 21 in the second data set, at times ranging from 7 months to 19 years after first presenting. Four spontaneous remissions occurred more than 10 years after initial onset of ITP. In total, 39 children with chronic ITP have thus far had a spontaneous remission. Many of those with chronic ITP from the earlier data set have now been lost to follow up, because they were asymptomatic with only mild thrombocytopenia, or have moved elsewhere since data on their status were collected in 1982. There are sufficient follow up data on 59 of the 66 cases from data set 1 , and on all 26 in data set 2 , to allow construction of a relatively crude curve showing the probability of spontaneous remission (figure). The predicted spontaneous remission rate is $61 \%$ after 15 years.

All in whom splenectomy failed remain under intermittent follow up. None has significant symptoms. All have platelet counts that on occasions exceed $40 \times 10^{9 / 1}$ and some occasional counts of $>100 \times 10^{9} / 1$. One splenectomised patient developed a brain tumour (see below). In total, 68 of the 92 children $(74 \%$; $95 \%$ confidence interval 64 to $82 \%$ ) are thus far known to have had remissions.

The indications for splenectomy in the five recent cases were prolonged, symptomatic thrombocytopenia, epistaxes, and educational problems in two children, prolonged steroid administration with concurrent hypertension requiring $\beta$ blockade in one, symptomatic thrombocytopenia and failure of azathioprine in one and repeated, prolonged steroid treatment in the fifth. Splenectomy was carried out 18 months after initial presentation in one, one year after in three, and six months after in another. In a sixth case, splenectomy is planned because of steroid refractoriness.

\section{OUTCOME RELATED TO DURATION OF INITIAL} SYMPTOMS

The table shows the relationship between duration of initial symptoms and outcome. Children with chronic ITP whose presenting symptoms were of more than two weeks' duration were less likely to be cured by splenectomy $(p<0 \cdot 03)$. All 12 with short histories were cured. Only one child suffered a true relapse after an initially successful splenectomy. This child, previously reported, ${ }^{7}$ did not have a detectable accessory spleen and is included among those in whom

Outcome in relation to duration of presenting symptoms

\begin{tabular}{|c|c|c|c|c|}
\hline \multirow[b]{2}{*}{$\begin{array}{l}\text { Duration of } \\
\text { symptoms }\end{array}$} & \multicolumn{2}{|c|}{ Splenectomy } & \multicolumn{2}{|c|}{ Failed or no splenectomy } \\
\hline & Remission & $\begin{array}{l}\text { No } \\
\text { remission }\end{array}$ & $\begin{array}{l}\text { Spontaneous } \\
\text { remission }\end{array}$ & $\begin{array}{l}\text { Persisting } \\
\text { ITP }\end{array}$ \\
\hline $\begin{array}{l}\leqslant 2 \text { Weeks } \\
>2 \text { Weeks }\end{array}$ & $\begin{array}{l}12 \\
17\end{array}$ & $\begin{array}{l}0 \\
8\end{array}$ & $\begin{array}{l}19 \\
20\end{array}$ & $\begin{array}{r}7 \\
17\end{array}$ \\
\hline & \multicolumn{2}{|c|}{$\mathrm{p}<0.03$} & \multicolumn{2}{|c|}{$p=0.21$} \\
\hline
\end{tabular}


splenectomy failed. The trend towards less frequent spontaneous remission in those with a longer, perhaps more insidious, period of bruising is not statistically significant $(p=0 \cdot 21)$.

\section{DEATHS}

Three of the deaths in the first data set have already been reported, ${ }^{6}$ and the lack of direct relevance of chronic ITP itself emphasised ${ }^{8}$; one died of drowning several years after a spontaneous remission, one in a road traffic accident, and one from pneumococcal sepsis after splenectomy. One further patient is known to have died of a malignant thalamic astrocytoma more than 20 years after his original presentation. In the second data set one boy, whose chronic ITP developed after Hodgkin's disease, died from an intracranial haemorrhage after a physical assault to his head. No unprovoked haemorrhagic death, solely attributable to chronic ITP, has occurred in either group. The single septic death occurred before the risks of postsplenectomy sepsis were known. Since regular penicillin prophylaxis and, later, immunisation against pneumococcal infection have been employed, no case of postsplenectomy sepsis has occurred but too few splenectomies have been carried out for meaningful analysis.

\section{EFFECTS OF TREATMENT OTHER THAN SPLENECTOMY}

Prednisone was used at some stage of the disease at least as frequently (in 25 of 26 cases) in the more recent group as in the earlier cases (21 of 66 ), for whom it was available after 1962. In the more recent group 10 children were prescribed several or prolonged courses of prednisone. Spontaneous recovery occurred promptly in one and improvement in another on stopping it. There is no suggestion that steroid treatment was of more than temporary benefit. Azathioprine, interferon alfa, and vitamin $\mathrm{C}$ were used without benefit in one child each. Intravenous IgG infusions were used in nine of the 26 recent cases but induced remission in none. From the point of diagnosis of chronic ITP (six months after initial presentation) 14 children from the recent group received no specific treatment. Lack of intervention may even have been beneficial as nine of the 11 spontaneous remissions thus far observed in the second data set occurred in these 14 children.

\section{Discussion}

This study, which combines recently diagnosed cases with those in the previous report, ${ }^{6}$ is essentially retrospective. It will probably have missed occasional cases, most likely some mildly affected children who had spontaneous remissions or adolescents not referred to a paediatric haematologist. None the less, these 92 children comprise the largest study of chronic ITP in children to have been reported, and for some purposes it is reasonable to amalgamate the two groups of children. Attempts to illustrate in detail, and over the entire time course during which the cases arose, the likely outcome in all those remaining at risk of spontaneous remission are hampered by the fact that several cases from the first data set have been lost to follow up and by lack of knowledge about the precise timing of remission in some. Despite these problems, the predicted spontaneous remission rate in the 85 children for whom there are reasonable data is $61 \%$ after 15 years, and in total 39 children are known to have had a spontaneous remission. Thus this study's major importance lies in confirmation of the high spontaneous remission rate suggested in several small studies ${ }^{9-12}$ but it also documents the low mortality and fails to show long term benefit from active treatment other than splenectomy.

Few reports of ITP in children focus on chronic disease, partly due to its rarity. Lilleyman estimates its incidence as perhaps one per 250000 children per year. ${ }^{3}$ From the comparatively small, recent data set in this study the observed minimum incidence is similar: $0 \cdot 46 / 10^{5}(1 / 220000)$ per year. Although Walker and Walker certainly included in their series most recognised cases of chronic ITP arising from Northumberland, Durham and what are now Tyne and Wear, Cumbria and Cleveland, ${ }^{6}$ the fact that the study was restricted to the experience of a single centre precludes calculations of incidence from their data. Despite this, their rate of accrual $(2 \cdot 2$ cases per year over 30 years) is only slightly below that ( $2 \cdot 6$ per year) of the second, population based data set. If this calculated incidence is correct and has not altered over time, approximately 12 cases of chronic ITP within the region were missed in their series. The combined groups of patients probably represent close to $90 \%$ of all childhood chronic ITP arising during 40 years within the Northern health region.

Walker and Walker's observation of a link between duration of presenting symptoms and eventual outcome has remained useful in the general approach to newly presenting children with ITP, 6 but its potential relevance to the management of chronic ITP, as it is now defined, was not examined. Reanalysis of their data, when combined with the observed outcome of more recently diagnosed cases, shows that children with a short initial history who develop chronic disease have a significantly greater chance of remitting after splenectomy than those with longer histories. Potential bias due to selected referral of more severe cases in the earlier group could be a confounding influence if those more mildly affected cases who recovered spontaneously (and were not referred) were more likely to have had prolonged initial histories. This is implausible. It is possible, however, that adult physicians and general haematologists only rarely referred adolescents with chronic ITP to paediatricians in the 1950 s and 1960s. Their behaviour would not have been documented. There is also a trend towards increased chance of spontaneous remission in those with short histories. This trend, however, is too weak at present to be clinically valuable and should in any case be interpreted with caution because some children 
who remitted after splenectomy (and not included in the crudely defined group at risk of spontaneous remission) did not have their operation until more than one year after initial presentation. The two week cut off in duration of presenting symptoms is arbitrary, as is the current definition of chronicity, but there may be underlying biological differences between those with a sudden and others with an insidious onset of ITP. This study was not designed to investigate that possibility nor to look for a more meaningful definition of chronic ITP. Whatever the biological implications, the link between duration of presenting symptoms and outcome of splenectomy in those who develop chronic ITP may be at least as useful as platelet sequestration studies, ${ }^{13}$ as yet not adequately evaluated in children, when assessing the potential role of splenectomy in an individual child.

Of the five deaths, two were accidental and one from an unrelated tumour. Two children would probably not have died had they not had chronic ITP but death was a direct complication of splenectomy in one and the result of a physical assault in another. It will need a study at least an order of magnitude greater than the 40 years' accumulated experience of chronic ITP from this region accurately to estimate its mortality and to distinguish iatrogenic influences from those of the disease itself. No case can be made from these data that splenectomy can reduce the risk of death.

Decisions about splenectomy are unique to each case and involve many factors, some unquantifiable, which affect the child's quality of life and the extent of the disability. They are also influenced by the perceived risks of the procedure. Splenectomy's fall from favour, a result of growing awareness of these risks, was suggested in the earlier report. ${ }^{6}$ It was carried out only half as frequently in the more recent group. It is possible that its frequency in the earlier group was artificially high because of central referral of more serious cases. The similarity in rates of accrual in the two groups suggests that a selection effect, if present, was not great. The data in this report support recently expressed opinions ${ }^{2414}$ that splenectomy should not be entered into hurriedly and may contribute to further decline in its popularity.

Despite the wide range of possible therapeutic approaches, vinca alkaloids, anti-D, and danazol have not been favoured in this region. Interferon alfa and azathioprine are rarely employed. Some paediatricians/haematologists use more prednisone and intravenous IgG than others, and appear to prefer repeated or prolonged courses of either than to accept modest or fluctuating thrombocytopenia, but without detectable long term benefit. In one case splenectomy was preferable to steroid induced hypertension. Improvement in at least two children in the more recent group directly followed cessation of steroid treatment, a pattern observed previously by Walker and Walker. ${ }^{6}$ However, all those cases were treated before management guidelines were published. ${ }^{4}$

Data from this study concerning the incidence, outcome, and trends in management of chronic ITP will supplement the copious published opinions. Natural history remains less clear because most children received some form of treatment, even if only within the first six months after first presenting with ITP. However, many children from both groups have been followed up for long periods, once chronic ITP was established, without specific treatment. Their behaviour is likely to be as close to the natural history of this disease as it will be possible to observe. This study has shown that chronic ITP in children has a very low mortality, that splenectomy was the only active treatment to result in long term benefit and that the overall outcome, whether a reflection of natural history or the result of therapeutic intervention, is generally benign. Should splenectomy need to be seriously considered in an individual child, the length of presenting symptoms may influence the decision. This report should encourage those who wish to pursue a relatively non-interventionist plan of management in individual children. However, it does not help to identify the very rare child with chronic ITP who will have an unprovoked intracranial haemorrhage, not least because such a catastrophe has not yet been observed in the Northern health region.

I thank all the haematologists and paediatricians within the Northern health region who helped me compile this record of chronic ITP, Professor W Walker for stimulating my interest in it management, and Professor J S Lilleyman for helpful comments.

1 Lilleyman JS. Management of childhood idiopathic thrombocytopenic purpura. Br f Haematol 1983; 54: 11-4.

2 Chessells J. Chronic idiopathic thrombocytopenic purpura primum non nocere. Arch Dis Child 1989; 64: 1326-8. 3 Lilleyman JS. Chronic idiopathic thrombocytopenic purpura; the paediatrician's dilemma. Pediatr Hematol Oncol 1990; 7: 115-8.

4 Eden OB, Lilleyman JS. Guidelines for management of idiopathic thrombocytopenic purpura. Arch Dis Child 1992 67: $1056-8$.

5 Lilleyman JS. Definitions and dogma in childhood idiopathic thrombocytopenic purpura. Pediatr Hematol Oncol 1993; 10: xi-xiv.

6 Walker RW, Walker W. Idiopathic thrombocytopenia initial illness and long term follow up. Arch Dis Child 1984; 59: 316-22.

7 Reid MM, Saunders PWG, Fenwick JD, Walker W. Splenunculectomy in thrombocytopenic purpura. Arch Dis Child 1986; 61: 192-4.

8 Reid MM. Splenectomy and chronic ITP in children. $\mathrm{Br} \mathcal{F}$ Haematol 1992; 82: 481-2.

9 Ramos ME, Newman AJ, Gross S. Chronic thrombocytopenia in childhood. $\mathcal{F}$ Pediatr 1978; 92: 584-6.

10 Choi SI, McClure PD. Idiopathic thrombocytopenic purpura in childhood. Can Med Assoc $\mathcal{F}$ 1967; 97: 562-8.

11 Lamni AT, Lovric VA. Idiopathic thrombocytopenic purpura: an epidemiologic study. 7 Pediatr 1973 ; 83: 31-6.

12 Hoyle C, Darbyshire P, Eden OB. Idiopathic thrombocytopenia in childhood: the Edinburgh experience 1962-82. Scott Med ₹ 1986; 31: 174-9.

13 Najean Y, Dufour V, Rain JD, et al. The site of platelet destruction in thrombocytopenic purpura as a predictive index of the efficacy of splenectomy. Br f Haematol 1991; 79: $271-6$.

14 Tait RC, Evans DIK. Late spontaneous recovery of chronic thrombocytopenia. Arch Dis Child 1993; 68: 680-1.

\section{Addendum}

Since this study was completed, a single centre series of 100 children with chronic ITP has been reported (Aronis S, Platokouki H, Mitsika A, et al. Seventeen years of experience with chronic idiopathic thrombocytopenic purpura in childhood. Is therapy always better? Pediatr Hematol Oncol 1994; 11: 487-98) which suggests a similarly high rate of spontaneous remissions. 